\title{
La temperatura de la intercara arrabio- refractario como variable determinante de los mecanismos de corrosión del crisol del horno alto
}

\author{
L.F. Verdeja ${ }^{(*)}$, P. Rusek ${ }^{(* *)}$, A. Alfonso ${ }^{(*)}$ y R. González ${ }^{(* *)}$
}

\begin{abstract}
Resumen El desgaste de materiales en el horno alto puede realizarse bajo la consideración de mecanismos estrictamente abrasivos o con el predominio de las componentes químicas y erosivas resultantes de la circulación de fluidos sobre el refractario. No obstante, aunque en determinadas situaciones, la abrasión o las componentes químicas pueden resultar los mecanismos controlantes, es necesario considerar que todos ellos (abrasión, erosión, desgaste químico, adhesión y desgaste térmico-choque térmico) "cooperan" en la degradación del refractario del horno. Para cualquiera de los mecanismos que interverga en el desgaste de los materiales, resulta conveniente conocer el valor de la temperatura a lo largo de las intercaras del refractario con los sólidos, líquidos o gases presentes en el horno. En la ponencia será desarrollada de forma específica, la importancia que tiene la temperatura en la intercara refractario-arrabio sobre el desgaste de los materiales del crisol. Se discutirán las diferentes hipótesis de transporte (energía, cantidad de movimiento y materia) más adecuadas para la obtención de la temperatura de intercara.
\end{abstract}

Palabras clave: Horno Alto. Crisol. Desgaste. Refractario.

\section{The temperature of the boundary pig iron/refractory as variable to determine the corrosion mechanism of the blast furnace hearth}

\begin{abstract}
The wear of materials in the blast furnace could be carried out under the consideration of abrasion mechanisms or with the prevalence of the components chemistries and erosives resulting as of the circulation of flowing on the refractory. Nevertheless, although in determining situations the abrasion or the components chemistries could result the control mechanism, it is necessary to consider, that all the abrasion, erosion, chemical corrosion, adhesion and thermal waste-thermal shock "they cooperate" to the degradation of the refractory of blast furnace. For whatever the mechanisms that intervene in the wear of the materials, it results interesting to know the temperature boundary of the refractory with the solids, liquids or present gases in the furnace. In the report, it will be developed from specific form, the importance that has the temperature in the boundary refractory-pig iron on the wear of the materials of the hearth. The different hypothesis of transport [energy, quantity of movement and material] more adequate for the obtaining of the boundary temperatures will be discussed.
\end{abstract}

Keywords: Blast furnace. Hearth. Wear. Refractory.

\section{INTRODUCCIÓN}

En la Siderurgia Integral, las mejoras alcanzadas en productividad y costes en los últimos tiempos

(*) Cátedra de Siderurgia. E.T.S.I. de Minas. Univ. de Oviedo. Independencia 13. 33004-Oviedo (España).

(**) Escuela de Ingeniería Industrial. Laboratorio de Mecánica. Universidad Panamericana. Augusto Rodin 498. Insurgentes Mixcoac. 03920-México D.F. (México). han ido paralelas al desarrollo de nuevos diseños y materiales en la construcción de elementos como el crisol del horno alto.

El éxito económico de un horno alto de gran capacidad (producción diaria superior a las 6000 t de arrabio) es consecuencia de una marcha altamente regular, sin que puedan interferir más interrupciones que las programadas. 
Recientemente, se han publicado informes de la Unión Europea sobre los criterios a seguir con el propósito de alargar lo más posible la campaña del horno alto (1 y 2). Básicamente, se puede afirmar que para lograr crisoles de altas prestaciones se debe actuar no sólo sobre la tecnología de materiales y diseño de las instalaciones, sino que también se tendrán que considerar los efectos de las variables operativas del horno:

- Refrigeración en paredes y fondo del crisol.

- Materiales de baja porosidad, alta resistencia en caliente y elevada conductividad térmica.

- Uniformidad en la distribución de gases reductores a lo largo de la carga.

- Características físicas de la zona de coque inactivo (hombre muerto), factor condicionante de velocidades de colada del arrabio (3 y 4).

- Número mínimo de ciclos de parada-arranque del horno.

- Procurar que durante las operaciones de colada del horno no llegue a salir gas por la piquera.

Dado que en las condiciones normales de trabajo de un horno no es posible realizar una medida directa del desgaste en paredes y fondo, se ha recurrido:

- A la formulación de modelos físicos en frío (2-4).

- Al control del desgaste del crisol mediante la introducción de sondas que midan la temperatura o el flujo de calor en determinadas cotas (2-5).

La solución de los problemas térmicos e hidrodinámicos del crisol, se traduce por el momento, a la aportación de unos mapas de isotermas o de líneas de corriente a lo largo de la geometría del crisol, resultado de la solución de ecuaciones diferenciales en derivadas parciales por métodos numéricos (FEM).

Se empiezan a conocer datos sobre los perfiles de desgaste del crisol al final de la campaña. Para crisoles monofásicos de bloques carbonosos, con refrigeración en paredes y fondo, el perfil más común es el que tiende a adoptar la huella de pie de elefante.

No existe, a la fecha, ninguna aportación que sustancie de forma cualitativa o semicuantitativa el mecanismo de desgaste de los materiales carbonosos, oxídicos y nitrurados en contacto con el arrabio. Igualmente no existe ninguna hipótesis que pueda explicar el perfil de desgaste que adopte el crisol al final de la campaña. Las aportaciones conocidas por la aplicación de simulaciones térmicas se traducen en la representación, al inicio de la campaña, de los perfiles de temperaturas a lo largo de la geometría del crisol en el plano (2D) o en el espacio (3D).
Con el Modelo de Desgaste Nodal (6 y 7), se pretende alcanzar un conocimiento tanto cualitativo como cuantitativo de los procesos de corrosión de los refractarios por fluidos agresivos (metales, escorias o gases), haciendo especial hincapié en el conocimiento y determinación de la temperatura en la intercara fluido-refractario.

\section{CONSIDERACIONES TEÓRICAS}

Estableciendo que alrededor de cualquier intercara sólido-fluido existe una zona de espesor variable (capa límite) a lo largo de la cual se desarrollan los perfiles de concentración, $\delta(C)$, temperatura, $\delta(T)$, y velocidades, $\delta(v)$, el Modelo de Desgaste Nodal sugiere como se tienen que calcular las distintas capas límites (9-11):

$$
\delta(v)=25\left(\frac{\mu}{\rho}\right) \frac{1}{\langle v\rangle}
$$

donde $\mu$ es la viscosidad del fluido y $\rho$, su densidad

$$
\delta(T)=\delta(v) P_{r}^{-(1 / 3)}
$$

en donde $P_{r}$, es el número de Prandtl.

$$
\delta(C)=2,25 D^{2 / 3}\left(\frac{\mu}{\rho}\right)^{1 / 3} \frac{1}{\langle v\rangle}
$$

donde $D$ es el coeficiente de difusión de la especie transportada, o bien $\delta(C)$ puede estimarse por la expresión:

$$
\delta(C)=0,97 S_{c}^{-(2 / 3)} \delta(v)
$$

donde $S_{c}$, es el número de Schmidt.

La determinación de la velocidad media, $\langle V\rangle$, dentro de la capa límite presenta dos componentes:

$$
<V>=V(f)+V(C)
$$

donde $V(f)$ es la componente de flotación (fuerzas de convección natural) y $V(C)$ es la correspondiente a las fuerzas de convección forzada (que pueden manifestarse cuando tiene lugar la colada del arrabio).

Aun cuando se pudiera estar realizando la colada del crisol, sobre todo en hornos de gran tamaño (diámetros superiores a los once metros), existen zonas del crisol en las cuales podemos considerar que $V(C)=0$. No obstante, es preciso reconocer que sería necesario avanzar en el conocimiento de 
los modelos hidrodinámicos del crisol del horno para poder concretar cuantitativamente el peso de este factor en la corrosión química de los refractarios.

La determinación de $V(f)$ en $\mathrm{m} / \mathrm{s}$, según el Modelo de Desgaste Nodal, se realiza mediante la ecuación:

$$
V(f)=6.98 \mu^{1 / 3} \rho^{-2 / 3} \Delta \rho^{1 / 3}(\mathrm{~m} / \mathrm{s})
$$

donde $\Delta \rho$ es la diferencia de densidad del fluido entre dos puntos-nodos de la intercara refractario-fluido que se calcula conociendo la temperatura en cada uno de estos puntos (6).

Para conocer la distribución a lo largo de la intercara arrabio-refractario, es necesario determinar cuál es el valor del coeficiente convectivo de transporte de calor en el arrabio, $h$, que depende de los valores que adopte el espesor de la capa límite térmica, $\delta(T)$. Por lo tanto, partiendo de un valor de $h$, se puede estimar el perfil de temperaturas y el espesor del desarrollo de las capas límites, a partir de las cuales, se tiene que calcular, cuál es el nuevo valor de $h$. El problema se encuentra resuelto cuando los valores estimados y calculados para $h$ sean lo más próximos posibles.

Para una situación en la cual únicamente se consideran la componente de flotación, $V(f)$, es el valor del coeficiente de transporte convectivo del arrabio igual a $85 \mathrm{~W} / \mathrm{m}^{2}{ }^{\circ} \mathrm{C}$. Es decir, pensando en valores de conductividad térmica efectiva (8) se tiene que, si la constante de transporte de calor por conducción en el arrabio a $1.500{ }^{\circ} \mathrm{C}$ es de $25 \mathrm{~W} / \mathrm{m}^{\circ} \mathrm{C}$ :

$$
\begin{gathered}
\beta=\frac{85 \mathrm{w} / \mathrm{m}^{\circ} \mathrm{C}}{25 \mathrm{w} / \mathrm{m}^{\circ} \mathrm{C}}=3,4 \mathrm{~m}^{-1} \\
h_{\text {(efectiva) }}=\beta K_{(\text {arrabio) }}
\end{gathered}
$$

donde $\beta$ es un coeficiente de proporcionalidad entre el coeficiente de transporte convectivo, $h$, y de conducción de calor, $K$, en el arrabio.

\section{RESULTADOS EXPERIMENTALES}

Cuantitativamente, los desgastes obtenidos para crisoles construidos por bloques carbonosos de alta conductividad media $\left(K=12 \mathrm{~W} / \mathrm{m}^{\circ} \mathrm{C}\right.$ y densidad global de $1.850 \mathrm{~kg} / \mathrm{m}^{3}$ ) muestran altas tasas de erosión anual, en aquellas zonas de la pared y del fondo próximas a las esquinas, mientras que en la zona central del fondo y de la pared del crisol se obtienen valores mínimos.

No obstante, los valores de máxima erosión (entre 29 y $41 \mathrm{~cm} / a n ̃ o)$ indican que el desgaste químico del crisol, sería importante en los inicios de la cam- paña y desaparecería prácticamente cuando los valores de la temperatura en la intercara se tornaran incompatibles con la termodinámica de disolución del carbono en el arrabio. En este instante, se bloquearía el mecanismo de desgaste químico, pero entraría en acción un mecanismo de desgaste suave abrasivo del crisol, tal y como proponen en una comunicación privada los Profs. Rusek y González de la Universidad Panamericana-México (1997). Puede pensarse que la erosión podría realizarse a través del carbono precipitado en la capa límite (a la temperatura del nodo, no es posible alcanzar un 4,50\% $\mathrm{C}$ disuelto en el hierro) que se desplazaría tangencialmente sobre los bloques de carbono con una velocidad expresada por la ec. [5].

\section{DISCUSIÓN Y CONCLUSIONES}

De la resolución de problemas térmicos, aplicando el Modelo de Desgaste Nodal, se llega al conocimiento cualitativo (temperaturas de intercara) y semicuantitativo (velocidades de erosión) que el desgaste, al menos durante las primeras fases de la vida del crisol, se debe fundamentalmente a mecanismos de desgaste de naturaleza química.

Los valores establecidos para materiales carbonosos en zonas de máximo desgaste han de corregirse a la baja debido a las siguientes consideraciones:

- Se ha tomado para todo el arrabio una $h$ (efectiva) que no es la misma a lo largo de la intercara arrabio-carbón, ya que la $h$ (efectiva) es una función de la dimensión lineal característica $\delta(T)$, presente en el número de Grashof utilizado en el cálculo del coeficiente de transporte convectivo natural de calor.

- En la solubilidad del carbono en el arrabio influye negativamente la presencia de silicio, manganeso, fósforo y azufre (parámetros de interacción).

El Modelo de Desgaste Nodal explica la existencia de zonas de máximo y mínimo desgaste en un crisol carbonoso, tal y como se ponen de manifiesto en los perfiles de desgaste conocidos al final de las campañas. Igualmente, justifica la influencia favorable, que tanto las condiciones de refrigeración como la conductividad térmica de los bloques de carbón, tienen sobre la velocidad de corrosión química del crisol.

La solución conocida como "taza cerámica" aplicada al diseño de crisoles de horno alto instalados en los años 90 analizada desde los planteamientos desarrollados por el Modelo de Desgaste Nodal puede resultar interesante, ya que indicaría que, durante los primeros años de la vida del horno, actuarían preferentemente los mecanismos de desgaste 
químicos en materiales sílico-aluminosos de alta o media alúmina, hasta lograr un perfil de temperaturas en la intercara arrabio-refractario que impida termodinámicamente la corrosión química del grafito microporoso, de medio-alto valor y presentar, a la abrasión suave de las partículas carbonosas, como la responsable del desgaste del crisol hasta el final de la campaña.

\section{Agradecimiento}

Los autores del trabajo quieren agradecer al CICYT-MAT97-1184 la financiación concedida para el desarrollo del proyecto.

\section{REFERENCIAS}

(1) JAMESON, D., LUNGEN, H. y LAO, D. Technical study into the means of prolonging blast furnace campaingn life. Contrato No 7210-ZZ/570. European Commission, 1995.

(2) PicARd, M. y TÉmoin, F. Amélioration de la durée de vie du haut fourneau, par la maîtrise des pertes thermiques. Contrato N $\mathrm{N}^{\circ}$ 7210-AA-313. European Commission, 1990.
(3) Schulz, V. y RÜTHER, H. Untersuchungen zur roheisenströmung im hochofengestell und abstichoptimierung, insbesondere zur verschleibminderung und vermeidung, von durchbrüchen. Contrato $N^{\circ} 7210-\mathrm{AB} / 112$. European Commission, 1993.

(4) Steiler, J., Venturini, M., Leprince, G., Guenard, C., Gauje, P., Dufour, A. y Libralesso, J. Etude thermique et hydrodynamique du creuset du haut-fourneau. Contrato $\mathrm{N}^{\circ}$ 7210-AA/314. European Commission, 1991.

(5) Schulte, M., Klima, R., Ringel, D. y Voss, M. Stahl Eisen, 117 (11), 1997: 57-63.

(6) Verdeja, L.F., Alfonso, A., Gonzalez, R. y BarranZuEla, J.L. Rev. Minas 13-14, 1996: 109-117.

(7) Verdeja, L.F., Alfonso, A., Rusek, P. y Barranzuela, J.L. V Encuentro del Hemisferio Sur sobre Tecnología Mineral. Buenos Aires, 1997: 341-344.

(8) Acutti, C. Rev. Metal. Madrid. 33 (5), 1997: 333-344.

(9) Chapman, A.J. Heat Transfer. $3^{\text {rd }}$. Collier Macmillan Intern. Edition, 1974.

(10) Polrier, D.R. y Geiger, G.H. Transport phenomena in materials processing. TMS Publ. 1994: 226.

(11) Deo, B. y Boom, R. Fundamentals of steelmaking metallurgy. Prentice Hall Intern. 1993: 114. 